\section{Collecting interstellar dust grains}

Interstellar dust grains are swept into the Solar System by the motion of the Sun through the interstellar medium. The velocity of interstellar grains as they enter the Earth's atmosphere is modulated by the Earth's motion around the Sun, reaching a minimum when the Earth is moving in the opposite direction to the Sun. Modelling of the entry heating in that season indicates that some of the interstellar dust particles enter the Earth's atmosphere without vaporizing, and that they could be retrieved from the Earth's stratosphere in the same way as the interplanetary dust particles that are collected by NASA aircraft.

Ion trails of dust particles have been detected $^{1}$ entering the Earth's atmosphere at velocities that significantly exceed the Solar System escape velocity, identifying these particles as interstellar. There is a seasonal variation in the flux, the peak flux probably indicating the time of year when the Earth, in its heliocentric orbit, is moving towards a stream of interstellar particles. The direction of movement increases their geocentric velocity so that particles as small as $15 \mu \mathrm{m}$ produce detectable meteors $^{1}$. Six months later, when the Earth is moving away from the stream, the ecliptic component of the particle's geocentric velocity is reduced by twice the Earth's heliocentric velocity of $30 \mathrm{~km} \mathrm{~s}^{-1}$, so that at the two extremes the atmospheric entry velocities are given by:

$$
v_{\text {entry }}=\left((v \sin i)^{2}+(v \cos i \pm 30)^{2}\right)^{1 / 2}
$$

where $i$ is the inclination angle, and $v$ is the heliocentric velocity of the particle at the time of entry.

Taylor et al. ${ }^{1}$ indicate that most of the interstellar dust is in a stream (their stream A), which has an atmospheric entry velocity of about $90 \mathrm{~km} \mathrm{~s}^{-1}$ on day 32 , the peak of the flux, and which is inclined at roughly $30^{\circ}$ to the ecliptic plane. Six months later, these particles enter the Earth's atmosphere at a velocity of approximately $39.6 \mathrm{~km} \mathrm{~s}^{-1}$.

I modelled the peak temperature reached by a dust particle entering the Earth's atmosphere using the methods described in refs 2 and 3. This correctly predicted that interplanetary dust particles would survive atmospheric entry. Interstellar grains of diameter 15-40 $\mu \mathrm{m}$ have been detected striking the Earth ${ }^{\text {, }}$, so I calculated the distribution of peak temperatures for $15 \mu \mathrm{m}$ grains of four densities $(0.5,1.0$, 2.0 and $4.0 \mathrm{~g} \mathrm{~cm}^{-3}$ ), spanning the range from porous interstellar aggregates of silicate and carbonaceous material ${ }^{4}$ to compact circumstellar grains extracted from meteorites $^{5}$ (Table 1).

For an entry velocity of $39.6 \mathrm{~km} \mathrm{~s}^{-1}$, all of the 15- $\mu \mathrm{m}$-diameter particles of density $0.5 \mathrm{~g} \mathrm{~cm}^{-3}, 21 \%$ of the particles of density $1 \mathrm{~g} \mathrm{~cm}^{-3}$, and $5 \%$ of the particles of density $2 \mathrm{~g} \mathrm{~cm}^{-3}$ reach peak temperatures below $1,900 \mathrm{~K}$ - the melting point of typical extraterrestrial silicates. Although interstellar silicates smaller than $15 \mu \mathrm{m}$ in diameter would not produce detectable radar signals, the observation of interstellar grains of diameter 15-45 $\mu \mathrm{m}$ (ref. 1) indicates that smaller grains might also be present. If so, these grains would be heated less severely than the $15 \mu \mathrm{m}$ grains modelled, and grains in the 5-10 $\mu \mathrm{m}$ size range would have a significantly higher probability of surviving entry unaltered.

A stream of 0.5 - $\mu \mathrm{m}$-diameter interstellar grains, with a velocity of approximately $26 \mathrm{~km} \mathrm{~s}^{-1}$, was detected at a distance of roughly 5.0 astronomical units (AU) from the Sun by the Ulysses spacecraft $^{6}$. Modelling indicates that, at some times in the solar cycle, these particles can reach the Earth ${ }^{7}$. Assuming the same $30^{\circ}$ inclination to the ecliptic, these $0.5 \mu \mathrm{m}$ interstellar grains should enter the Earth's atmosphere at roughly $29 \mathrm{~km} \mathrm{~s}^{-1}$ when the Earth is moving away from the stream.

To model the peak temperature reached by $0.5 \mu \mathrm{m}$ grains on atmospheric entry requires an assumption of the thermal emissivity. It has been shown ${ }^{8}$ that the

\begin{tabular}{|c|c|c|c|c|c|c|c|c|}
\hline \multicolumn{2}{|c|}{ Particle } & \multicolumn{7}{|c|}{ Fraction of incident particles not heated above temperature } \\
\hline $\begin{array}{l}\text { Diameter } \\
(\mu \mathrm{m})\end{array}$ & $\begin{array}{l}\text { Density } \\
\left(\mathrm{g} \mathrm{cm}^{-3}\right)\end{array}$ & $800 K$ & $1,000 \mathrm{~K}$ & $1,200 \mathrm{~K}$ & $1,400 \mathrm{~K}$ & $1,600 \mathrm{~K}$ & $1,800 \mathrm{~K}$ & $1,900 \mathrm{~K}$ \\
\hline 15.0 & 0.5 & $8.9 \times 10^{-4}$ & $5.2 \times 10^{-3}$ & $2.2 \times 10^{-2}$ & $7.6 \times 10^{-2}$ & $2.2 \times 10^{-1}$ & $5.7 \times 10^{-1}$ & 1.0 \\
\hline 15.0 & 1.0 & $2.1 \times 10^{-4}$ & $1.2 \times 10^{-3}$ & $5.3 \times 10^{-3}$ & $1.8 \times 10^{-2}$ & $5.3 \times 10^{-2}$ & $1.4 \times 10^{-1}$ & $2.1 \times 10^{-1}$ \\
\hline $\begin{array}{r}15.0 \\
\ldots 4 \ldots \ldots\end{array}$ & 2.0 & $5.2 \times 10^{-5}$ & $\begin{array}{l}3.1 \times 10^{-4} \\
.\end{array}$ & $1.3 \times 10^{-3}$ & $4.6 \times 10^{-3}$ & $1.3 \times 10^{-2}$ & $3.4 \times 10^{-2}$ & $5.2 \times 10^{-2}$ \\
\hline 15.0 & 4.0 & $1.3 \times 10^{-5}$ & $7.7 \times 10^{-5}$ & $3.3 \times 10^{-4}$ & $1.1 \times 10^{-3}$ & $3.5 \times 10^{-3}$ & $8.9 \times 10^{-3}$ & $1.4 \times 10^{-2}$ \\
\hline 0.5 & 1.0 & $1.2 \times 10^{-2}$ & $7.5 \times 10^{-2}$ & $3.2 \times 10^{-1}$ & 1.0 & 1.0 & 1.0 & 1.0 \\
\hline 0.5 & 3.0 & $1.3 \times 10^{-3}$ & $8.1 \times 10^{-3}$ & $3.4 \times 10^{-2}$ & $1.2 \times 10^{-1}$ & $3.4 \times 10^{-1}$ & $8.9 \times 10^{-1}$ & 1.0 \\
\hline
\end{tabular}

emissivity for olivine spheres declines from near one, as assumed in the Whipple model $^{2}$, to approximately 0.2 as the size of the particle decreases to $5 \mu \mathrm{m}$. Assuming a lower emissivity of 0.1 for the $0.5 \mu \mathrm{m}$ interstellar grains, the peak temperatures reached are much lower than for the $15 \mu \mathrm{m}$ grains (see Table 1).

Thus, significant fractions of both the large interstellar grains detected by Taylor et $a{ }^{1}{ }^{1}$ and the smaller grains detected by the Ulysses spacecraft ${ }^{6}$ are expected to survive atmospheric entry when the Earth is moving away from the interstellar dust stream. During this season, the interstellar grains will settle into the Earth's stratosphere in the same manner as the interplanetary dust particles routinely collected by NASA sampling aircraft. Interstellar particles in the size range 5-20 $\mu \mathrm{m}$ may be present on NASA collection surfaces that are already flown, but redesigned collectors would be required for the efficient collection of the $0.5 \mu \mathrm{m}$ particles.

Convincing identification of an individual particle as interstellar would come from detection of non-solar isotopic compositions of either the refractory elements (for example oxygen or silicon) or the trapped noble gases, both of which have been used to identify interstellar grains from meteorites ${ }^{5}$. However, some interstellar grains are likely to have solar isotopic ratios. These might be identified by their extreme radiation damage ${ }^{9}$ or by observing the seasonal variation in particles having chemical compositions and structures consistent with those inferred for interstellar dust. This would provide laboratory samples of the most abundant dust of the interstellar medium rather than just the atypical grains isolated from meteorites.

\section{G. J. Flynn}

\section{Department of Physics,}

State University of New York,

101 Broad Street, Plattsburgh,

New York 12901-2681, USA

\footnotetext{
Taylor, A. D. et al. Nature 380, 323-325 (1996).

2. Whipple, F. L. Proc. Natl Acad. Sci. USA 36, 687-695 (1950)

3. Fraundorf, P. Geophys. Res. Lett. 19, 765-768 (1980).

4. Greenberg, J. M. \& Hage, J. I. Astron. Astrophys. 286, 915-924 (1994)

5. Zinner, E. Science 271, 41-42 (1996).

6. Grun, E. et al. Astron. Astrophys. 286, 915-924 (1994).

Gustafson, B. A. S. \& Lederer, S. M. in Physics, Chemistry and Dynamics of Interplanetary Dust, ASP Conf. Ser. V 104, 35-38 (Astron. Soc. Pacific, San Francisco, 1996).

8. Rizk, B. et al. J. Geophys. Res. 96, 1303-1314 (1991).

9. Bradley, J. P. Science 265, 925-929 (1994).
}

scientific correspondence is intended to provide a forum in which readers may raise points of a scientific character. Priority will be given to letters of fewer than 500 words and 10 references. 